\title{
SATTLER'S VEIL
}

BX

\author{
J. Dallos
}

LON DON

IN my report to the Széchenyi Scientific Society in 1934 I gave a detailed account of experiments made with contact lenses fitted so as to correspond, or on the contrary to deviate systematically from casts taken from the eyeball. My conclusion at that time was that the best average tolerance, i.e., the longest periods of wear without irritation or veiling, is reached when a lens fits firmly over large areas in the upper-inner and lower-outer quadrants, not with edges but surface to surface, and has apart from these primarily fitting areas a secondary touch, i.e., contact without pressure, spreading over the middle of the cornea; the centr: pait of the lens should have a radius somewhat flatter than the cornea so that an air bubble can freely circulate around the limbus and escape in the lacrymal meridian (from up and out to down and in) which is made comparatively loose, thus allowing a certain amount of " breathing."

I emphasised that this applies only if the fitting is judged in terms of actual behaviour. For taking a cast and altering the mould so as to conform statically to the above requirements will as a rule not result in a lens that fits as expected, and further alterations, dependent on the accuracy of the cast and on the morphology of the eye (configuration of the eyeball, thickness and compressibility of the conjunctiva, lid pressure, etc.) are necessary to prevent rotation and dislocation and thus to produce a dynamically balanced fit which promises satisfactory wear.

In the following years evenness and steadiness of fit gained in importance, and eventually it was clear that for mere physical comfort nothing equals an even fitting such as I advocated ir: Oxford in 1937. If a lens is made so as to touch the eye evenly over the cornea and the bulbar conjunctiva with a gentle clearance towards scleral and limbal margins, comfort is immediate and lasting. Care must be taken, however, to assure that what appears to be an even fit really is an even fit. Thus for instance the loss through grinding out a capillary layer of glass in the centre raising it by $50 \mu$, should completely sever contact with the cornea; scleral sectors must not only touch evenly but also help their neighbours and their counterparts in the opposite half of the same meridian-for no meridian, not even the horizontal, is ever in full view in its entire length-to keep an equal, even proximity to the surface of the eye. 
This, of course, can neither be reached nor confirmed, except by actually altering the inside of the lens, grinding out some areas and propping up others with capillary layers of wax. Individual fitting today still means a series of adjustments immediately proved right or wrong by trial and error. Judging the fit correctly is helped by the behaviour of conjunctival vessels as well as by the route rather than the presence of air bubbles whilst the lens, after dry insertion, is touched home.

If the scleral sectors are well balanced a horizontal mark on the lens will keep its position, rotating some degrees only when the eye moves towards extreme positions (mostly up and in), but returning to its original line when looking straight again. Such a lens will follow the moving eye with less than $1 \mathrm{~mm}$. lag, without showing blanching areas, and without letting an air bubble slip in from the periphery.

To reach a balanced, even fit is sometimes easy. More often it means painstaking, exacting work, sometimes exhausting the time and patience of the prospective wearer to a degree. In fact, a number of final lenses had to be made during the war years, when time was limited and carrying out the work was imperative, which were necessarily a compromise between "theoretical", requirements and practical usefulness. Complaints arising out of this have been invariably put right by finishing the fitting to routine accuracy.

The last ten years' work at the Contact Lens Centre brought conclusive evidence as to the existence of a well defined type of fitting that gives maximum comfort beyond doubt; moreover, there is sufficient proof (over 2,000 cases) that given the time this type of fitting can be attained in every case, whatever pathological conditions prevail (trephine scars, cystic scars after extraction, symblepharon bridges, scarred atrophy in delayed mustard gas keratitis, advanced stages of trachoma and pemphigus, etc.).

Whilst workers were pre-occupied with the difficulties of producing contact lenses which should be both tolerable and optically corrective-fighting all the time the suspicion and bias of the medical world - the minor drawback of a slight blur which develops only after several hours of wear, remained wholly in the background. For people who wear contact lenses for periods of two hours or more (sometimes less) will, as a rule, develop a corneal haze very similar to that accompanying a mild attack of glaucoma. Under the slit-lamp a faint epithelial oedema is seen, the patient notices a slight bluish haze in daylight and coloured haloes around lights in the dark. After removing the lens the condition clears in 20-30 minutes. 
Described as early as 1889 by A. Muller, who noticed the phenomenon in his own eye, it was not until the International Congress in Amsterdam, 1929, and the launching of Zeiss's larger set of contact lenses by Heine that an interest in contact lenses was taken up on a larger scale and that the frequency and constancy of the "veil" was observed by all. My first intimation of any definite opinion as to its nature (oedema) and seat (corneal epithelium only) was in 1934 by personal communication from Prof. C. H. Sattler of Koenigsberg. Hence the name.

Sattler's veil, although the commonest change in the corneal epithelium is by no means the only form of epithelial oedema that contact lenses may cause. More pronounced changes were first observed by Fick in 1887 in the rabbit. In man the swollen epithelium may assume a stippled or wrinkled surface; the fitted pattern of the former shows an array of tiny air bubbles over areas in contact with the smooth lens, suggesting that each pit is an imprint of a tightly captured minute air bubble. Wrinkles, particularly when covered by a large air bubble behind a lens fitting loosely in the centre give the impression as if the cornea had dried out. Sattler's veil, on the other hand, is even and macroscopically diffuse. As a rule it starts quite imperceptibly all over the cornea, but in some cases it may begin in an odd sector somewhere in the periphery, spreading in irregularly coalescent streaks towards and past the centre. Also, if the epithelium is allowed to swell further; particularly in some morbid cases, the oedema will collect in vesicles of various sizes, but clustering over one preferential area. Loss of corneal epithelium, if it ever occurs after removing a contact lens worn too long, i.e., over two hours after onset of veiling, is the result of burst vesicles and not of suction and tear.

Any permanent damage I have seen in the last fourteen years with evenly fitted contact lenses was the consequence of secondary infection of epithelial lesions of this kind in persons who grossly ignored the veil (one street trader with conical corneae who had not the intelligence and one jungle fighter who had not the chance to remove the lenses in time, also a high myope who would not be discouraged from over-wearing his contact lenses).

As to the aetiology, it is rather obvious that some change in the physiological functions of the cornea caused by the continued presence of the contact lens must be responsible. One school of thought stressed " internal suffocation," interference with the blood circulation, strangulation of the limbal mesh that provides the nutritional fluids for the cornea and of the small veins of the ciliary region that help to carry away toxic products of metabolism. Others thought that, at least in this latter respect, some extra ocular 
factors like stoppage of tear circulation, prevention of the constant massage of the eye by the lids (blinking) an'd occlusion of the cornea from the air, are of greater importance.

There are many arguments against the "outer suffocation" theory. Surely, we do not wake with a veil after a sound night's sleep. Airtight bandages and Lohnstein chambers can be worn for hours on end without any corneal haze developing. And there is plenty of scope for tear circulation under any evenly fitted contact lens (if inserted with 5 per cent. argyrol, the brown tinge turns yellow and vanishes within minutes), yet the veil will appear inexorably after a number of hours.

The length of clear wear, i.e., the initial period between insertion of the contact lens and onset of the veil, shows considerable individual variations and tends to confuse the issue rather than clarify it. In the first stages of wear it usually takes 2-3 hours for the veil to. appear, but in some cases of supposedly reduced vitality, like extreme myopes, aphakic eyes with cystic scars, extensive post-operative or inflammatory scarrings in the limbal area, etc., it may be as little as 40 minutes; and it may take over an hour to clear away. The veiling may be different in the two eyes of the same individual, in aphakics this is rather the rule than the exception. On the other hand, in some cases, particularly in some very young and fit people, it may be very long indeed before the veil appears. In a 19-year-old service-man, who once wore his lenses for nine days and nights without removing them, the veil was perceptible at the end of the day, but disappeared overnight. In cases of reduced corneal sensitivity the veil is often too slight to notice.

Apart from these individual variations the clear periods show a regular increase with regular wear, reaching a limit within 3-8 months; in constant wearers this will amount to 8-14 hours per day if split up into two approximately equal periods with a 1-2 hours' rest in between. It is then not unusual to find a marked decrease in the tension of the eyelids; I shall come back to this presently.

Lacking biological foundation, measures to influence the veil were for a long time few and empirical, if any. Experiments with Zeiss's spherical sets in the early 1930 -s seemed to show that lenses that sit on the cornea cause less veiling than those with a space between. However, spherical lenses of a tighter corneal fit are very irritating and too trying to wear for long enough for the veil to appear.

With the advent of individual fitting, this early rule of more irritation-less veil, less irritation-more veil, seems to have 
changed to the contrary; on the same eye an evenly fitted contact lens will cause earlier veiling when the eye is irritable. Clumsy insertion, inclusion of a rubbing eyelash, smoky atmosphere, a hot room, reading at too close range, will cause avoidable irritation and precipitate an early veiling, whereas open air on a dull, cool day will keep off both. Earlier veiling, after all, seems to go hand in hand with discomfort rather than the other way round.

The fact that a loosely worn, comfortable contact lens allows a longer span of clarity, that insensitive or hypo-aesthetic corneae hardly veil, and that increasing use makes for decreasing veiling, all point to the tension of the eyelids as playing an important rôle in pressing the lens so tightly on to the surface of the eye as to interfere seriously with some of its physiological functions. Although I suspected this many years ago (Hung. Ophthal. Soc., 1931) it was F. A. Williamson-Noble who first succeeded in obtaining decisive experimental proof. In some stubborn veilers Williamson-Noble blocked the facial nerve with proctocaine and found a considerable extension of clear wear. As long as the akinesia lasted, and in at least one case much longer, the veil never appeared. But there were some failures, too, one veiling in 40 minutes while both eyelids were still quite paretic. Also I felt that a facial block was too heavy a price to pay for wearing contact lenses. I decided therefore to resume some of my early experiments.

First I wished to find out to what extent I could relieve the conjunctiva from pressure by more conservative means. Then I wanted to go into the possible merits of the " outer suffocation " theory championed by F. P. Fischer.

Accordingly some cases with good tolerance but early veiling were tried again with lenses that fitted so as to make more use of a corneal fulcrum. In some cases veiling was appreciably postponed (in one high myope clear periods of three hours were extended to six hours, making a daily total of 12 ), but in many others the improvement was less obvious and, as expected, increased irritability often prevented longer periods of satisfactory wear.

For experiments with free air circulation I picked two patients, one aphakic (R.E. 4 hrs., L.E. cystic scar, $45 \mathrm{~min}$. veiling) and one myope ( 2 hrs. R. \& L.). First I inserted a contact lens with a $14 \mathrm{~mm}$. opening in the middle, a sort of individually shaped scleral ring. This did not give rise to a veii at all. Subsequently the opening was reduced so that the lid no longer touched the middle of the cornea whilst blinking. Still, there was no veil. Further reduction of the hole seemed to defeat 
its purpose by getting blocked whether it was cornea or conjunctiva that lay behind it, and the veil appeared again.

However, by careful adjustment the lenses were finally made to fit so as to keep up air circulation through one or more active perforations and yet remain in constant contact over the pupillary area.

This was reached by making the inside curvature grossly flatter than that of the cornea $(e . g ., 8.5 \mathrm{~mm}$. radius if that of the cornea is $8.0 \mathrm{~mm}$.), thus providing ample air space around the limbus where the lens is subsequently perforated. Perforations under the upper lid are more cosmetic but less efficacious, partly because the lid itself blocks the way of free diffusion, and partly because sinking of the lens by gravitation places the cornea in an upward eccentric position thus blocking free access to the circling air bubble.

Since these two cases were fitted there has been hardly enough time to experiment on a large scale, but the absence of veiling has been invariable in all the cases I have since fitted on these lines.

Apart from the absence of veiling, there is another striking feature, i.e., the absence of the initial irritation. So far it was usual to find that in the initial stages twenty to twenty-five minutes after insertion a certain amount of irritation developed which was immediately relieved by detaching the lens from off its seat (" loosening," lifting the lens off the eye by hooking the fingernail behind the outer edge whilst looking nasally). Most people overcame this trouble in the course of regular wear, and it was generally assumed that the cause of the phenomenon was mechanical irritation through suction precipitated and aggravated by the tension of untrained eyelids. It now appears that this irritation was not mechanical at all, but chemical, probably due to the gradual rise of the $\mathrm{pH}$ as the $\mathrm{CO}_{2}$ concentration increased. Any additional factor that contributed to quicker production of $\mathrm{CO}_{2}$, including hyperaemia due to mechanical irritation and to increased sealing off of the pre-corneal space (tighter scleral fit) was thus capable of hastening the appearance of the veil. It looks as though not only Sattler's veil is clearing, but also the one which obscured our knowledge of its aetiology.

From the foregoing it would appear that the principles of fitting have come back full circle with " breathing" again in the foreground, but with a difference; whereas previously breathing was achieved through an unbalanced fit, thus causing mechanical irritation which more than outweighed chemical comfort, it is now possible to introduce free air circulation into a lens with an even balanced fit.

The new type of fitting is by no means without additional 
difficulties. What is most bewildering for the fitter is the complete absence of suction, with the lens actually falling out of the eye as the upper lid is lifted. As regards the patient, the optical effect of peripheral air bubbles is hardly perceptible and, if so, easily overcome, but holes showing in the palpebral aperture are not aesthetic although a perforated contact lens in a comfortable white eye is much less conspicuous than an unperforated one in an irritable eye however slight the irritation that precedes and accompanies the veil.

However, once there is a working principle, I am confident that ways will be found, perhaps through a combination of air pockets and channels, and holes where they are not visible (porous substances would probably have a limited delaying action only, like buffer solutions), to overcome this last minor trouble with contact lenses.

I would like to take this opportunity of thanking Mr. F. A. Williamson-Noble for his kindness in allowing me to make use of material from his hitherto unpublished experiments. For permission to refer to their cases I wish to acknowledge my indebtedness to Lady Duke-Elder, Mr. A. Rugg-Gunn, Mr. P. G. Doyne and Mr. T. G. Wynne Parry, and in particular to Professor Ida Mann for her invaluable co-operation over many years.

Technical facilities were generously placed at my disposal by Messrs. Theodore Hamblin, Ltd.

\title{
CONJUNCTIVAL FINDINGS IN CASES OF COLD HAEMAGGLUTINATION*
}

\author{
BY \\ SÜREYYA GÖRDÜREN \\ ANKARA
}

THE investigation of conjunctival blood circulation in cases of cold haemagglutination, where capillary and blood changes occur, is very important.

Before the following details I will explain some peculiarities of conjunctival blood circulation. Conjunctival vessels contrasting with the bright colour of scleral tunic can be clearly seen with the naked eye. When we use slit-lamp microscopy it becomes the ideal place for capillaroscopy.

* Received for publication, April 11, 1946. 